\title{
ESTUDO PRELIMINAR DE INDICADORES DE QUALIDADE DE UM CURSO DE GRADUAÇÃO EM ENGENHARIA QUÍMICA
}

\section{PRELIMINARY STUDY OF QUALITY INDICATORS OF AN UNDERGRADUATE COURSE IN CHEMICAL ENGINEERING}

Lucas Geőcze Cotrim¹, Danilo Magalhães Assunção do Carmo² e Antonio Marcos de Oliveira Siqueira ${ }^{3}$

1 Universidade Federal de Viçosa, UFV, Viçosa/MG, Brasil, E-mail: $\frac{\text { lucasgcotrim@gmail.com }}{2}$ Universidade Federal de Viçosa, UFV, Viçosa/MG, Brasil, E-mail: 2 Universidade Federal de Viçosa, UFV, Viçosa/MG, Brasil, E-mail: danilo.magalhaesac@gmail.com 3 Universidade Federal de Viçosa, UFV, Viçosa/MG, Brasil, E-mail: antonio.siqueira@ufv.br, ORCID: https://orcid.org/0000-0001-9334-0394

\section{AR T I C LE INFO}

Article history:

Received 2020-09-07

Accepted 2020-12-12

Available online 2020-12-12
Palavras-chave: Projeto Pedagógico. Matriz Curricular. Engenharia Química.

Keywords: Pedagogical project. Curriculum. Chemical engineering.

ABSTRACT. In view of the implementation scenario of the new National Curricular Guidelines - DCNs-, the present work proposes to study on the indicators of the Chemical Engineering course at the Federal University of Viçosa (UFV). A study was carried out of the proposals presented by the new resolution, as well as a survey of performance quality indicators for undergraduate courses in Brazil. It was possible to contextualize the Chemical Engineering course through the critical analysis of the indicators raised, as well as indicating ways in which the preparation of a new pedagogical project for the course can meet the objectives of the current DCNs, seeking to promote greater multidisciplinary and transdisciplinary in their pedagogical practice, with a greater emphasis on the development of students' skills.

RESUMO. Diante do cenário de implementação das novas Diretrizes Curriculares Nacionais - DCNs-, o presente trabalho se propõe a fazer um estudo sobre os indicadores do curso de Engenharia Química da Universidade Federal de Viçosa (UFV). Para tanto, foi realizado um estudo das propostas apresentadas pela nova resolução, bem como um levantamento de indicadores de qualidade de desempenho dos cursos de graduação no Brasil. Com o estudo, foi possível contextualizar o curso de Engenharia Química por meio da análise crítica dos indicadores levantados, bem como indicar caminhos pelos quais a confecção de um novo projeto pedagógico do curso possa atender aos objetivos das DCNs vigentes, buscando promover maior multidisciplinaridade e transdisciplinaridade em sua prática pedagógica, com maior ênfase no desenvolvimento de competências dos graduandos. 


\section{INTRODUÇÃO}

As matrizes curriculares dos cursos de engenharia química foram regidas até 2019 pela Resolução CNE/CES 11/2002, que apresenta as Diretrizes Curriculares Nacionais do Curso de Graduação em Engenharia (DCN's). A graduação em engenharia deve possuir um projeto político pedagógico do curso (PPC) que apresente o processo evolutivo de aprendizagem do discente ao longo da graduação, as habilidades e competência que serão desenvolvidas no estudantes, como se dará o processo de ensino-aprendizagem e quais as diretrizes para garantir a formação de um egresso qualificado para as demandas econômicas, sociais e ambientais, prezando pelo desenvolvimento sustentável nesse tripé (BRASIL, CNE, 2002).

Em abril de 2019, institui-se a Resolução CNE/CES 04/2019, que reformula as Diretrizes Curriculares Nacionais de um Curso de Graduação em Engenharia assegurando a formação de novos engenheiros que seja mais conectada com o tripé econômico-social-ambiental demandada na segunda e terceira década do séc XXI. Esta Resolução define que os cursos terão até abril de 2022 para adequarem-se às novas Diretrizes e reformulem suas propostas políticas pedagógicas consequentemente (BRASIL, CNE, 2019).

O presente trabalho constitui-se de um estudo preliminar do curso de Engenharia Química da Universidade Federal de Viçosa (ENQ UFV), utilizando-se de dados de qualidade de ensino disponibilizados pelo Ministério da Educação, Folha de São Paulo (RUF. FOLHA DE SÃO PAULO, 2019) e Guia do Estudante (EDITORA ABRIL, 2017). Diante do cenário de implementação das novas Diretrizes Curriculares Nacionais - DCNs-, faz-se necessário uma análise do contexto da engenharia química da Universidade Federal de Viçosa, sua evolução ao longo dos anos, o estado em que se encontra atualmente e de quais formas o curso precisa se adequar para atender às novas Diretrizes, que serão obrigatórias a partir de abril de 2022 . O trabalho considera aspectos como a prática pedagógica de cursos de engenharia no Brasil (NITZ, et al., 2018), bem como um paralelo com o contexto da prática pedagógica da engenharia química da Universidade Federal de Viçosa.

\section{REVISÃO DA LITERATURA}

Amorim (2016) realizou uma investigação a respeito dos projetos pedagógicos dos cursos de engenharia De uma instituição federal, dirigida à identificação de problemas em certos aspectos dos textos desses documentos, com ênfase para a concepção de tecnologia aplicada a processos produtivos, o foco na formação direcionada ao mercado/empresas e o perfil esperado dos profissionais, bem como para tratamento dado a disciplinas de ciências humanas em termos de discurso. Buscou-se fazer considerações sobre o caráter de formação do engenheiro, tendo como premissa que sua formação não pode limitar-se apenas aos fatores técnicos da profissão. O resultado dessa investigação indicou que tal noção se faz presente ao longo dos textos analisados, ao mesmo tempo em que se constata a forte influência de concepções mais tradicionais. De um modo geral, ainda se observa a predominância de uma concepção mais voltada à formação de profissionais para o mercado de trabalho.

Ao menos num ponto de vista presente nos textos dos projetos pedagógicos, entende-se que eles se dedicam a formação de um egresso cujo perfil é condizente 
com o explicitado pela Resolução CNE/CES 11/2002, numa concepção de que o egresso deve atender as demandas da sociedade com base na resolução de problemas. $O$ trabalho ainda ressalta a necessidade de consolidação da prática da pesquisa, organizada em equipes multidisciplinares, voltada a compreensão dos contextos sociais de atuação, para a identificação de desafios a serem superados e promoção do diálogo interdisciplinar.

A respeito da visão dos discentes e egressos de cursos de engenharia investigados acerca do perfil desenvolvido no decorrer da prática pedagógica, Silva et al. (2019) buscaram obter dados quantitativos que permitissem uma avaliação de seu nível de satisfação. Um dos objetivos de tal trabalho foi a apresentação de um modelo que pudesse servir para pesquisas de satisfação em outras universidades, abordando ainda aspectos avaliativos como os instrumentos de avaliação do MEC e a inserção no mercado de trabalho. Sua abordagem obteve um resultado interessante ao demonstrar que é comum encontrar contrastes entre cursos avaliados positivamente pelos instrumentos do MEC e resultados de baixa satisfação entre os egressos, o que aponta para a necessidade de uma maior atenção de coordenadores de curso e de gestores do ensino para indicadores de satisfação do aluno, contribuindo para processos de tomada de decisão que visem a garantia da excelência da prática de formação acadêmica e profissional.

Pinto e Pinto (2019) fazem uma série de comentários acerca da situação dos cursos de engenharia diante do marco de 17 anos da publicação da Resolução CNE/CES 11/2002, ao avaliar que as diretrizes curriculares por ela estabelecidas não foram implementadas integralmente, e ainda classifica o cenário atual como um de poucos avanços da prática pedagógica em relação ao anterior à sua publicação. Em seu parecer, há um predomínio na realidade brasileira da utilização de projetos pedagógicos de caráter tradicional, defasados do contexto contemporâneo, no que se constata como uma baixa interdisciplinaridade entre os componentes curriculares. Dessa forma, as novas diretrizes curriculares instituídas possuem por objetivo o fortalecimento de práticas pedagógicas que favoreçam essa integração. O ponto fundamental apresentado pela nova Resolução é a possibilidade dada às instituições de ensino superior (IES) de refletirem sobre suas práticas, o que permite a elaboração de PPC construídos de forma mais coerente e coletiva, não se limitando a um grupo restrito de docentes. De um modo geral, o trabalho ressalta a grande necessidade de inovações na formação dos engenheiros no Brasil.

Ferlin (2019) fez uma análise dos currículos de cursos de engenharia do período 1998-2018, comparando suas situações de cumprimento da carga horária estabelecida pelas diretrizes curriculares vigentes nesse período. Em sua avaliação, é importante que a elaboração estrutural de uma matriz curricular e de um projeto pedagógico do curso (PPC) tenham como base o instituído pelas diretrizes curriculares para garantir o atendimento do estabelecido no perfil do egresso. Entretanto, levanta-se a problemática da falta de relação entre os elementos apresentados no texto do PPC e as ações efetivas da prática pedagógica, fazendo do PPC um documento cuja função efetiva é o cumprimento de exigências legais pertinentes ao ensino de engenharia. Portanto, entende-se que é preciso reforçar o estabelecimento de uma harmonia adequada entre os componentes curriculares, com o fim de promover uma maior dinâmica de saberes, bem como um desenvolvimento mais efetivo de habilidades e competências relacionadas não apenas à figura do profissional apto a lidar com aspectos técnicos, mas que também reflitam as expectativas de um cidadão comprometido com o exercício crítico de seu papel social.

Por sua vez, Dias e Grigol (2019) investigaram de forma qualitativa as DCNs com ênfase para as competências e habilidades, buscando entender sua relação com 
a formação do profissional de engenharia, numa perspectiva em que se faz uma reflexão sobre as necessidades de transformação curricular diante dos avanços tecnológicos contemporâneos, pertinentes à área de engenharia. Entende-se que o ensino de engenharia no Brasil possui desafios variados, que partem das expectativas para o perfil dos egressos, para passar também pelo acompanhamento pedagógico dos alunos. Dessa forma, faz-se necessária a problematização do processo de formação de engenheiros no Brasil, para cujo perfil é necessário, além da capacitação técnica, ter afinidade para o trabalho em equipes multidisciplinares, postura empreendedora e interesse pela pesquisa. Para tanto, é preciso pensar em metodologias de ensino e aprendizagem que busquem o desenvolvimento de visões sistêmicas e humanísticas nos discentes. Uma das dificuldades desse processo está ligada ao fato de que o conhecimento vem, de forma cada vez mais intensa, se transformando com os avanços científicos e tecnológicos, facilidade de acesso à informação, o que enseja um processo adaptativo constante das universidades.

Ao considerar aspectos como a heterogeneidade entre os integrantes do curso, percebe-se a importância do fomento à participação em projetos para a promoção da integração dos alunos, além de incentivar a realização de programas de atendimento pedagógico e psicossocial que auxiliem na permanência dos estudantes, dado que tal acompanhamento pode impactar positivamente em indicadores como taxa de evasão, o que se reflete na possibilidade de elaboração de PPCs que contenham estratégias que contribuam para a permanência dos alunos nos cursos. Um aspecto interessante é o incentivo a adoção de práticas que incentivem a formação continuada do docente, priorizando-se, inclusive, a capacitação para o exercício da docência. Além disso, torna-se evidente a necessidade de realização de projetos que, além de relacionar os conhecimentos parciais das disciplinas, tratem dos impactos sociais e ambientais da prática da engenharia.

Ribeiro e Siqueira (2018) analisaram uma amostra de 271 estudantes e exalunos do curso. Foi-se constatado que a Engenharia Química da UFV consegue promover uma boa atração e desenvolvimento da capacidade analítica de raciocínio lógico nos estudantes que são muito demandadas em processos seletivos, refletindo em profissionais de alto nível e com forte capacidade para resolver problemas complexos. A discrepância observada, porém, encontra-se no desenvolvimento de habilidades cognitivas e sociais (RIBEIRO; SIQUEIRA, 2018).

\section{DIRETRIZES CURRICULARES NACIONAIS}

Como apresentado pela Resolução CNE/CES 04/2019 (atual) e pela Resolução CNE/CES 11/200 (anterior) por Diretrizes Curriculares Nacionais entende-se o conjunto de normas que devem orientar a redação dos PPC's e o planejamento das ações em um curso de graduação. O processo de normatização é feito de modo a garantir a flexibilidade para adequação dos PPC's a contextos geográficos e temporais amplos, verificando-se a necessidade de melhoria contínua e atenção para as inovações pedagógicas e tecnológicas decorrentes da prática do ensino de engenharia. Portanto, depreende-se que a Resolução CNE/CES 04/2019, assim como a anterior, não apenas abre a possibilidade para esse tipo de ação, como também promove seu incentivo (BRASIL, CNE, 2019; BRASIL, CNE, 2002).

Uma das primeiras mudanças de concepção entre as duas novas resoluções diz respeito ao seu objetivo, ao acrescentar a avaliação e desenvolvimento de cursos de engenharia, o que aponta a necessidade de abordagem desses processos nos textos dos PPCs. Quando dispõe do perfil do egresso, constata-se que a nova 
Resolução apresenta uma concepção mais abrangente, com ênfase para o desenvolvimento da visão holística, reforçando a abordagem do ensino de Engenharia a partir de uma perspectiva global, e não regional. A nova Resolução ainda destaca a importância do desenvolvimento de um egresso com perfil inovador e empreendedor, o que se soma às noções prévias de predisposição para a pesquisa e desenvolvimento de novas tecnologias, explicitando a necessidade de soluções multidisciplinares e transdisciplinares para um contexto contemporâneo de problemas complexos. Aponta-se, ainda, a necessidade de atenção aos fatores humanos e sociais decorrentes da utilização da tecnologia, abarcando temas como cidadania e sustentabilidade (BRASIL, CNE, 2019; BRASIL, CNE, 2002).

Em seu artigo 4o, a nova Resolução trata do objetivo de desenvolvimento de competências e habilidades de forma mais detalhada que a anterior, além de reforçar a noção de que a formação do engenheiro deve se basear no oferecimento de conteúdos estruturados em sua matriz curricular (como ocorre tradicionalmente). Entretanto, a nova Resolução apresenta uma inovação conceitual ao determinar que o desenvolvimento de competências deve ser o objetivo da prática pedagógica, e não apenas consequência do fornecimento dos elementos necessários para o aporte do conhecimento. Isto implica numa forte necessidade de reformulação dos PPCs de modo a priorizar o desenvolvimento de competências nos alunos, ao invés de focar na estruturação do conteúdo. Outro aspecto interessante é ressaltar a necessidade de formação de um engenheiro que possa atuar como professor, portanto capaz de reproduzir tal processo de desenvolvimento de competências (BRASIL, CNE, 2019; BRASIL, CNE, 2002).

Acerca da nova estrutura dos PPCs (cuja instituição foi uma inovação da Resolução CNE/CES 11/2002), a nova resolução indica novos aspectos a serem contemplados. De acordo com Oliveira (2019) o PPC agora se situa em posição central para a organização do curso, ao definir os tópicos principais que devem ser desenvolvidos durante sua redação. A elaboração do PPC deve levar em conta aspectos da inserção geográfica do curso, as demandas do mercado e da sociedade desse contexto, bem como explicitar a trajetória completa do engenheiro em formação. Uma observação interessante acerca do cenário de inserção do curso é a atenção o fato de que os egressos só se formarão cinco anos após seu início, por isso quaisquer considerações a serem feitas sobre esse aspecto devem levar em conta não somente a situação presente, mas também as projeções futuras. Deve-se fazer também, de forma recorrente, a identificação das necessidades globais a serem atendidas pelos engenheiros, de modo a constantemente reestabelecer-se a amplitude da área de atuação da Engenharia. Além disso, é necessário um foco em questões da formação de engenheiros que pressuponham o acompanhamento destes enquanto estudantes, objetivando a identificação e o desenvolvimento de vocações individuais, bem como o combate à evasão, e dessa forma garantir que os objetivos estabelecidos pelo PPC estão sendo efetivamente alcançados. Isso é importante num contexto em que se observa, como consta no Parecer CNE/CES no 1, de 23 de janeiro de 2019, um aumento das taxas de evasão de cursos de Engenharia nos anos mais recentes, bem como uma redução da procura por esses cursos. Tal cenário pode estar, em boa medida, relacionado com o momento presente da economia brasileira, em que a indústria nacional passa por uma perda de competitividade se comparada a de outros países emergente, como pode-se constatar pela perda de participação do país no valor adicionado à produção de manufaturados, bem como baixo crescimento na participação das exportações de produtos industrializados.

Quando tratam das atividades que devem entremear a trajetória do estudante no curso, as novas diretrizes ampliam o que se dispunha na Resolução anterior, 
estimulando a adoção de práticas que combinem mutuamente a teoria, a atuação e seu respectivo contexto de aplicação. Reforça-se, também, a implementação de metodologias ativas de aprendizagem, mais alinhadas com o objetivo central da nova Resolução, o desenvolvimento de competências. A respeito dos conteúdos, a nova Resolução apenas apresenta os conteúdos básicos, sem dividi-los em núcleos (básico e profissionalizante), como proposto pela Resolução anterior.

\section{HABILIDADES E COMPETÊNCIAS}

De acordo com Catapan, Kassick e Otero (2016), o perfil do egresso se define por competências que podem ser divididas em três tipos: atitudinal, cognitiva e procedimentais-operacionais (habilidades). A competência atitudinal se dá pelo "ser" do indivíduo, em como este se porta diante das situações, evidenciando que questões éticas são consideradas em suas atitudes, no pensar da ação e das suas consequências, por exemplo, no âmbito socioambiental. A competência cognitiva diz respeito ao conhecimento e à capacidade de aplicar e relacionar diferentes conceitos de sua formação diante das situações que Ihes são apresentadas. Já por habilidade entende-se suas capacidades operacionais, pela capacidade de implementar as devidas ferramentas para lidar com o objeto profissional.

Portanto, o perfil profissional se apresenta como um conjunto de competências, divididas nessas referidas três dimensões: conhecimento, habilidades e atitudes, que englobam aspectos que se relacionam com o trabalho. Por conhecimento entende-se uma gama de informações apreendidas e estruturadas pelo indivíduo, resultado de sua experiência pessoal ao longo de seu tempo de vida. Já a habilidade se relaciona com o saber de como fazer uma determinada atividade, a uma capacidade de se colocar em prática determinado conhecimento, ou seja, implementá-los para se exercer uma função. Tal aptidão pode ser inata ou desenvolvida, e fatores como o treino e a prática possibilitam o aprimoramento individual (CHIAVENATO, 2014).

Cunha (2007) estabelece que habilidade consiste em dominar a utilização do intelecto para a execução de tarefas específica. A atitude, por sua vez, diz respeito a certos aspectos de caráter social e afetivo relacionados ao trabalho, indicando as predisposições que norteiam a adoção de uma ação específica, identificando-se padrões de recorrência, fazendo com que habilidade possa ser facilmente definida pelo conjunto de conhecimentos e atitudes (FERREIRA, 2010). Nesse sentido, apreende-se que a competência é a organização e associação dos conhecimentos por meio de mecanismos de caráter mentais, em contraste com as habilidades, ferramentas pelas quais a competência é colocada em prática (RAMOS, 2002). Os autores Green (2000); Spencer e Spencer (1993) estabelecem que competência por meio da observação dos comportamentos e resultados, uma vez que estes são passíveis da descrição objetiva, o que implica que é possível validar as causas de aumento de desempenho no trabalho por meio do comportamento. Complementa-se a essas noções a definição de Zarafian (2001), que trata como centro da competência a reação dos profissionais diante dos problemas complexos inerentes às situações de trabalho.

\section{ATIVIDADES COMPLEMENTARES NA ENGENHARIA}

Uma vez que a obtenção do conhecimento pode ser feita por meio do estudo, pela pesquisa e pela observação sistemática inerente a sua prática, tal conhecimento pode ser acumulável, constatando-se que a tecnologia é consequência desse capital 
de conhecimentos desenvolvidos pelo trabalho. Para tanto, torna-se fundamental a compreensão da visão sistêmica para o entendimento da educação organizacional continuada (MARIOTTI, 1999; PINHEIRO e BURINI, 2002).

Assim, o ensino superior tem como propósito mostrar aos discentes os principais aspectos da realidade presente na prática profissional da engenharia, como a complexidade das variáveis inerentes aos processos, as interações entre os diversos sistemas, as possibilidades de interações que ainda não foram observadas, as possibilidades de mudanças e os pontos principais de cada sistema. Portanto, para a efetividade da integração disciplinar, defende-se que, em geral, sejam organizados grupos de trabalho que fomentem o trabalho sinérgico dos seus membros, o que caracteriza tais grupos como equipes (MARIOTTI, 1999; PINHEIRO e BURINI, 2002).

De um modo geral, a prática de aprendizagem é feita por meio da fragmentação do conhecimento em várias disciplinas. Contudo, entende-se como essencial para o desenvolvimento da formação profissional a promoção da integração desses conhecimentos fragmentados. Portanto, é imperativo que o processo educacional se dê por meio de mecanismos que promovam a multidisciplinaridade, a interdisciplinaridade e a transdisciplinaridade. Multidisciplinaridade se dá quando várias disciplinas se agregam para a atuação no estudo de um assunto específico, sem que haja interrelacionamento. Assim, as disciplinas não interferem umas nas outras no decorrer da atividade. Já a interdisciplinaridade com a reunião e interação entre várias disciplinas, criando uma situação de integração mútua, o que permite a elaboração de uma síntese acerca das naturezas diversas de tais disciplinas. Por fim, entende-se por transdisciplinaridade uma integração interdisciplinar de modo que se verifica uma tendência à dissolução dos limites entre as disciplinas, permitindo que cada disciplina trate de aspectos das demais (PINHEIRO e VIEIRA, 2003).

\section{RESULTADOS E DISCUSSÃO}

No Ranking Universitário da Folha (RUF, 2019) a Engenharia Química UFV aparece como a $12^{\mathrm{a}}$ melhor do Brasil, sendo a $2^{\mathrm{a}}$ melhor em Minas Gerais. Observando a classificação da Universidade Federal de Viçosa como instituição, ela aparece em 15ำ no ranking de melhores Instituições de Ensino Superior do Brasil. Verifica-se que dentre os cursos da UFV que foram avaliados, destacam-se os cursos de agrárias como destaques no cenário nacional, com Agronomia sendo o $2^{0}$ melhor do país, Zootecnia o 3ํㅡㄹ melhor, Medicina Veterinária o 4ํㅡㄹ melhor do país. Tal resultado era esperado, visto que a Universidade Federal de Viçosa ocupa posição de destaque no cenário mundial nas áreas de agrárias e floresta e no ranking mundial de universidades QS Top Universities no período 2013 a 2019:

Quando se verifica, porém, a classificação dos cursos de exatas da UFV, destaca-se a Engenharia Química, criada em 2007, melhor engenharia da UFV e ocupando $012 \mathrm{o}$ lugar das melhores do Brasil, enquanto a engenharia mais antiga da UFV, criada em 1977, ocupa a posição 60 no ranking. Percebe-se, também, que o fator Avaliação de Mercado é o peso negativo da nota final. A Universidade Federal de Viçosa possui sua maior força na área de agrárias. Pode-se atribuir a isso o fato de a área das agrárias ser a mais antiga e mais consolidada da universidade, no oposto de que as engenharias foram majoritariamente criadas na primeira década do séc. XXI, ainda passando por uma fase atualmente de consolidação e estruturação. Viçosa é uma cidade que se encontra na região da Zona da Mata do estado de Minas Gerais, região onde há predominância de $73 \%$ de atuação de microempresas, dos segmentos de tecido, vestuário e calçados (SILVA, 2018). A configuração de mercado com a ausência de setor industrial desenvolvido na região é desfavorável para a 
atuação prática profissional do Engenheiro Químico, tendo uma relação direta com a baixa experiência dos estudantes de engenharia química da UFV em indústrias em estágios e projetos ao longo da graduação e, sendo provavelmente este o maior indício do motivo de uma nota de Avaliação de Mercado mediana. Por outro lado, a força do curso demonstrada pelo RUF está na Qualidade de Ensino. A ENQ UFV possui 46,88 pontos, de 64,00 possíveis, a colocando em 9 o lugar nesse quesito dentre todos os cursos analisados.

O Guia do Estudante faz uma análise qualitativa e quantitativa dos cursos de graduação no Brasil, mas divulga um resultado de Conceito Final do Curso qualitativo por meio das estrelas atribuídas. A ENQ UFV obteve três estrelas no ranking em uma escala de um (ruim) a cinco (excelente) e não se disponibiliza uma quebra desse Conceito final em subindicadores como no RUF, tornando-se assim dificultada uma análise mais detalhada a respeito de quais pontos foram os determinantes positiva e negativamente para a nota final atribuída.

Como parte do processo de análise de indicadores do curso de engenharia química internos à Universidades, uma entrevista com a Coordenação do Curso foi realizada no dia 25/09/2019, na sala da Coordenação no Departamento de Química e uma coleta de dados na Pró Reitoria de Ensino na mesma data. Percebe-se aqui que a situação do curso de Engenharia Química da UFV não desobedece a tendência já observada nos cursos de engenharia nacionais do ponto de vista global, e que pode muito bem estar relacionada com o cenário macroeconômico nacional, pois como consta do próprio PPC do curso, a região de Viçosa não compreende um parque industrial expressivo, naturalmente capaz de absorver a mão-de-obra egressa da universidade. Portanto, tal situação tornaria Viçosa dependente do cenário macroeconômico da indústria nacional, propondo-se como uma alternativa para a absorção de tal força especializada de trabalho o desenvolvimento da indústria regional, seja através de fábricas de pequeno, médio e grande porte.

O curso. O curso de Engenharia Química da Universidade Federal de Viçosa (UFV) iniciou-se em 2007 com oferta de 40 vagas anuais no campus UFV/Viçosa. O documento responsável por sua apresentação é o seu Projeto Político Pedagógico (PPC), caracterizado por sua natureza flexível, porém constituído em bases firmes que remetem às DCNs vigentes à época de sua redação, de modo a tornar possível que sua alteração seja feita de forma contínua, de acordo com os avanços constantes no campo educacional, bem como pelas mudanças nos cenários do desenvolvimento tecnológico, e pela própria natureza dinâmica das relações sociais, econômicas e ambientais em que o curso se insere. Numa única ocasião, como é de se esperar, o documento sofreu alterações em sua formulação original no ano de 2010, no caso para atualizar mudanças na matriz curricular do curso (UNIVERSIDADE FEDERAL DE VIÇOSA, 2010).

Informações da Estrutura dos Laboratórios do Curso. O curso de engenharia química da UFV possui dois laboratórios de ensino à sua disposição, um deles focado na área de processos, outro na área de operações (laboratórios 126 e 128 do Departamento de Química da UFV). Em geral, é no segundo que se encontram os módulos didáticos, porém os equipamentos podem ser transferidos entre os laboratórios a depender da necessidade. Os laboratórios também são utilizados para aulas dos cursos do Departamento de Tecnologia de Alimentos (DTA), para pesquisas de iniciação científica, TCC e pela pós-graduação em engenharia química. Ambos possuem área de $81,24 \mathrm{~m} 2$, com um espaço entre eles dividido em três seções, de $9,55,9,75$ e $9,55 \mathrm{~m}^{2}$. 
Corpo docente do curso. A Engenharia Química não possui departamento próprio, ficando subordinado ao Departamento de Química e com quadro reduzido de corpo docente. Todos os professores trabalham sob regime de dedicação exclusiva. O corpo docente da engenharia química UFV é bem enxuto, com os professores possuindo uma alta sobrecarga de trabalho em relação à quantidade de horas trabalhadas semanalmente. Os docentes dividem-se entre pesquisas em projetos e publicações científicas, apoio à empresa júnior, ao centro acadêmico, aos projetos de ensino e extensão e às 35 disciplinas da engenharia química do mestrado e da graduação. Esta configuração dificulta consideravelmente 0 desenvolvimento do curso, com por exemplo a criação de um doutorado vinculado ao curso de engenharia química e a própria evolução de carreira dos professores, que não conseguem sair para um pós-doutorado, por exemplo, por não haver quem os substituiria nas atividades ao longo do período acadêmico. Além dos professores acima listados, o corpo docente atual possui também dois professores substitutos com contratos temporários em regime de trabalho reduzido, com 40 horas semanais

Demais Indicadores do Curso. Os indicadores de que se dispõe hoje fornecem um parâmetro bom da qualidade do curso. Temos boas avaliações no ENADE e no Guia do Estudante. Temos também na UFV pós-graduação strictu sensu (mestrado). Mas não possuímos indicadores que meçam a empregabilidade dos egressos do curso, não sabemos em quais segmentos profissionais os alunos entram e em quais deles eles se desenvolvem mais. A Coordenação do Curso de engenharia química comentou a respeito da ausência de indicadores que mostrem o desempenho dos egressos no mercado de trabalho. Pesquisas científicas realizadas por alunos da engenharia química UFV, porém, dão uma perspectiva a respeito do cenário profissional dos egressos.

Para uma comparação mais consistente entre os diversos cursos de engenharia química no Brasil, indicadores de infraestrutura são necessários. Um comparativo da quantidade de laboratórios, quantidade de módulos de ensino em cada laboratório, a área do laboratório por aluno e quantidade de módulos de informática por aluno são exemplos de possíveis indicadores. O comparativo por infraestrutura daria uma noção mais precisa da qualidade proporcional de um curso em relação à estrutura que possui e ao repasse financeiro realizado pela universidade e governo. Seriam indicadores interessantes também a relação de professores por alunos, distribuição de bolsas de projetos para professores e alunos e indicadores que acompanhassem a evolução profissional dos egressos nos diversos setores, o que potencialmente contribuiria para o entendimento de quais setores do mercado mais absorvem os profissionais da universidade em análise.

\section{CONCLUSÕES INICIAIS}

Ao longo deste trabalho, foi possível contextualizar certos elementos do cenário em que se encontra o curso de Engenharia Química da UFV, 12 anos após sua criação. A avaliação da situação do curso diante de um cenário em que são publicadas novas diretrizes curriculares é importante para, além de identificar êxitos e pontos de melhoria, encontrar caminhos pelos quais a UFV pode se adequar às novas diretrizes, dado que elas trazem uma mudança de abordagem em relação ao desenvolvimento de competências e habilidades.

O levantamento de metodologias ativas, cujo foco se alinha justamente com o objetivo da nova Resolução, é uma forma de apresentar meios pelos quais a redação de um novo PPC pode tirar inspirações importantes, inclusive resgatando experiências do próprio curso de Engenharia Química da UFV. A utilização dos indicadores de 
avaliação do curso, tanto aqueles empregados pelo MEC, quanto os que são empregados pelos veículos de mídia de massa, uma vez interpretados de forma crítica, permite que se alcance um entendimento do contexto do curso através dos aspectos quantitativos e qualitativos que permeiam a criação desses indicadores.

O trabalho, portanto, propõe que a mudança de abordagem dos conteúdos por um viés com uma ênfase maior em aspectos de multidisciplinaridade e transdisciplinaridade é entendida como um fator essencial para nortear o processo de ensino-aprendizagem no contexto da UFV. Tal proposta é condizente com o propósito das novas diretrizes, dado que a própria noção de competências pressupõe a capacidade de conexão entre diferentes saberes. Deve-se atentar para o fato de que o PPC é um documento que, na ótica das novas DCN's, deve passar por um constante processo de releitura para a realização de diagnósticos acerca dos objetivos expostos em seu texto. Deve-se ressaltar que a experiência do próprio curso com a implementação de metodologias ativas precisa ser observada com a devida atenção, o que permite a obtenção de insumos práticos para a implementação de tais medidas. Sugere-se, ainda, que trabalhos posteriores procurem desenvolver novas práticas para implementação dessas metodologias, além de investigarem formas para a adoção das práticas previstas nas novas DCN's.

É preciso, ainda, reforçar que as limitações presentes do curso de Engenharia Química da UFV no que diz respeito a disponibilidade de recursos humanos e materiais impõe restrições para a capacidade de implementação efetiva de ações nas direções indicadas. Faz-se essencial a expansão das dependências físicas disponíveis para a prática pedagógica do curso, bem como a contratação de maior contingente de profissionais para a sua operacionalização.

\section{AGRADECIMENTOS}

Os autores agradecem o apoio por parte da Universidade Federal de Viçosa (UFV). O presente trabalho foi realizado com apoio da Coordenação de Aperfeiçoamento de Pessoal de Nível Superior - Brasil (CAPES) - Código de Financiamento 001 e da Fundação de Amparo à Pesquisa do Estado de Minas Gerais (FAPEMIG).

\section{REFERÊNCIAS}

Nitz M., Raffaelli, R.R.P., Bresolin, I.T.L, Tonso A., Camacho, J.L.P., Paiva, J.L., Le Roux, G.C. Distribuição e desempenho dos cursos de engenharia química no Brasil uma análise com base nos microdados do INEP de 2012 a 2016. COBEQ 2018. p. 4863-4866.

AMORIM, M. L. Qual engenheiro? - Uma análise dos Projetos Político-Pedagógicos do curso de engenharia química da Universidade Tecnológica Federal do Paraná (UTFPR). Revista de Ensino de Engenharia, v. 35, n. 1, p. 23-33, 2016.

BRASIL. CNE. Resolução CNE/CES 002 24/04/2019. Institui as Diretrizes Curriculares Nacionais do Curso de Graduação em Engenharia. Brasília, DF. Diário Oficial da União ํำ 80, 26.04.2019, Seção 1, p. 43.

BRASIL. CNE. Resolução CNE/CES 11/2002. Institui Diretrizes Curriculares Nacionais do Curso de Graduação em Engenharia. Diário Oficial da União, Brasília, DF, 9 de abril de 2002. Seção 1, pág. 32. 
Catapan, A. H.; Kassick, C. N.; Otero, W. R. I. Metodologia para elaboração de matriz curricular: integração e transversalidade. Unisul, Tubarão, v.10, n. Especial, pág. 27 45, jun/dez 2016.

Chiavenato, I. Gestão de pessoas (4. ed.). Rio de Janeiro: Elsevier. 2014.

Cunha, G. D. Diretrizes para a Elaboração de Projetos Pedagógicos de Cursos de Engenharia. Rio de Janeiro: Associação Brasileira de Engenharia de Produção, 2007.

Dias, R. D.; Grigol, R. B. Educação em engenharia: estudos curriculares e práticas pedagógicas inovadoras. XLVII COBENGE, Fortaleza, 17 a 20 set. 2019.

EDITORA ABRIL: Guia dos Estudantes Profissões 2018. São Paulo, SP, 2017. Disponível em: <https://guiadoestudante.abril.com.br/universidades/entenda-aavaliacao-de-cursos-2017-do-guia-do-estudante/>. Acesso em: 19/07/2020.

Ferlin, E. P. Análise do currículo de cursos de engenharia nos últimos 20 anos (1998 - 2018): estudo de caso nos cursos de engenharia da computação. XLVII COBENGE, Fortaleza, 17 a 20 set. 2019.

Ferreira, J. Aspectos relevantes na abordagem Comportamental. In A. Neri (Ed.). Gestão de $\mathrm{RH}$ por competências e a empregabilidade (5. ed.). Campinas: Papirus. 2010.

GREEN, P. C. Desenvolvendo competências consistentes. Rio de Janeiro: Qualitymark, 2000.

Mariotti, H. Organizações de Aprendizagem: Educação Continuada e a Empresa do Futuro. São Paulo: Atlas, p. 179, 1999.

Oliveira, V. F. As inovações nas atuais diretrizes para a Engenharia: estudo comparativo com as anteriores. A Engenharia e as Novas DCNs: Oportunidade para formar mais e melhores engenheiros. LTC, 2019.

Oliveira, A. S. D.; Pereira M. S.; Lima, L. M. Trabalho, Produtivismo e Adoecimento dos Docentes nas Universidades Públicas Brasileiras. Uberlândia, 2017. Revista Psicologia Escolar e Educacional, São Paulo, Volume 21, Número 3, setembro/dezembro de 2017.2 Disponível em: <http://www.scielo.br/pdf/pee/v21n3/2175-3539-pee-21-03-609.pdf>. Acesso em: 02/06/2020.

Pinheiro, A. C. F. B.; Burini, E. R. V. Uma Proposta de Aprendizado Baseado em Problemas para o Ensino Interdisciplinar nos Cursos de Administração. Encontro Nacional de escolas de administração - ENANGRAD, 2002, Rio de Janeiro. Anais do XIII ENANGRAD. Rio de Janeiro: ANGRAD, 2002.

Pinheiro, A. C. F. B.; Vieira, J. L. As atividades complementares nos cursos de engenharia como agentes integradores na formação profissional. COBENGE, São Paulo, 2003.

Pinto, D. P.; Pinto, M. S. Comentários sobre as novas diretrizes curriculares nacionais. XLVII COBENGE, Fortaleza, 17 a 20 set. 2019.

Ramos, M. N. A educação profissional pela pedagogia das competências e a superfície dos documentos oficiais. Education et Sociétés, p. 401-422. 2002.

Ribeiro, J. E. M.; Siqueira, A. M. de O. Fatores que influenciam a empregabilidade de recém-formados no mercado de trabalho. XLVI COBENGE, Salvador, 03 a 06 de 
setembro. 2018.

RUF. FOLHA DE SÃO PAULO: Ranking Universitário da Folha: Ranking de Cursos de Graduação. São Paulo, SP, 2019. Disponível em: $<$ https://ruf.folha.uol.com.br/2019/>. Acesso em: 10/05/2020.

Silva, C. G.; Mesquita, E. G.; Bahia, M. G. M.; Viana, M. L. S. Análise comparativa da satisfação entre discentes e egressos e engenharia: um estudo de caso. XLVII COBENGE, Fortaleza, 17 a 20 set. 2019.

Silva, E. L. O. Fecomércio MG apresenta dinâmica econômica da Zona da Mata. Belo Horizonte, MG, Brasil. 21/11/2018. Disponível em: $<$ http://www.fecomerciomg.org.br/2018/11/fecomercio-mg-apresenta-dinamicaeconomica-da-zona-da-mata/s. Acesso em: 25/06/2020.

Spencer, L. M.; Spencer, S. M. Competence work: models for superior performance. Nova York: John Wiley \& Sons, 1993.

UNIVERSIDADE FEDERAL DE VIÇOSA. Projeto Político Pedagógico do Curso de Engenharia Química da UFV. Viçosa, MG, 2010. Disponível em $<$ http://www.enq.ufv.br/arquivos/Projeto_Politico_Pedagogico.pdf>. Acesso em: 15/05/2020.

Zarafian, P. Objetivo por competência: por uma nova lógica. São Paulo: Atlas. 2001. 